\title{
ASA Fall 2019
}

\section{View Abstract}

\section{CONTROL ID: 3153592}

TITLE: Reverberation time and audibility in phased geometrical acoustics using plane or spherical wave reflection coefficients

AUTHORS (FIRST NAME, LAST NAME): Matthew Boucher ${ }^{1}$, Monika Rychtarikova ${ }^{2}$, Lukas Zelem³ ${ }^{3}$ Bert Pluymers ${ }^{4}$, Wim Desmet ${ }^{4}$

INSTITUTIONS (ALL): 1. Structural Acoustics Branch, NASA Langley Research Center, Hampton, VA, United States.

2. Faculty of Architecture, KU Leuven, Gent, Belgium

3. Faculty of Civil Engineering, Department of Architecture, STU Bratislava, Bratislava, Slovakia.

4. Mechanical Engineering, Division PMA, KU Leuven, Heverlee, Belgium.

\section{ABSTRACT BODY:}

Abstract (200 words): In acoustical spaces, room acoustics parameters are often predicted using energybased geometrical acoustics. For smaller rooms, interference among coherent reflections is taken into account by phased geometrical acoustics, which improves results for lower frequencies. The use of a spherical wave reflection coefficient improves the results further, yet the impact on room acoustics parameters is not fully known. This work focuses on the differences in predicted reverberation time when using plane or spherical wave reflection coefficients. The differences are analyzed for a variety of boundary conditions, including nonuniform distributions of absorption, in medium-sized rooms using a phased image source model. Since calculated differences are greater than the conventional just-noticeable-difference of $5 \%$ for reverberation time, a laboratory listening test is performed to confirm audibility of the modeled differences. Two narrow band noise stimuli (octave bands with central frequency 125 and $250 \mathrm{~Hz}$ ) with a duration of $1 \mathrm{~s}$ were used for comparisons of 18 acoustic scenarios by means of a three-alternative forced choice method (3AFC). More than half of the listeners could hear the differences in all 36 cases. Statistically significant results (chi-squared test was used) were found in two thirds of the cases, corresponding to those with longer reverberation times.

CURRENT TECHNICAL COMMITTEE: Architectural Acoustics

CURRENT SPECIAL SESSION. IF NOT SUBMITTING TO A SPECIAL SESSION, CHOOSE "NONE

SELECTED.": Room Acoustics Modeling and Auralization

PRESENTATION TYPE: Invited Submission : Lecture

PRESENTER: Matthew Boucher

AWARDS:

AV Equipment: (none)

AUTHORS (ADDRESS \& EMAIL):

Name: Matthew Boucher

Address 1: MS 463

Address 2: (none)

City: Hampton

State: VA

Postal Code/Zip: 23681-2199

Country: United States

E-mail: matthew.a.boucher@nasa.gov

Name: Monika Rychtarikova

Address 1: Hoogstraat 51

Address 2: (none) 
City: Gent

State: (none)

Postal Code/Zip: 9000

Country: Belgium

E-mail: Monika.Rychtarikova@kuleuven.be

Name: Lukas Zelem

Address 1: (none)

Address 2: (none)

City: Bratislava

State: (none)

Postal Code/Zip: (none)

Country: Slovakia

E-mail: lukas.zelem@stuba.sk

Name: Bert Pluymers

Address 1: (none)

Address 2: (none)

City: Heverlee

State: (none)

Postal Code/Zip: (none)

Country: Belgium

E-mail: bert.pluymers@kuleuven.be

Name: Wim Desmet

Address 1: (none)

Address 2: (none)

City: Heverlee

State: (none)

Postal Code/Zip: (none)

Country: Belgium

E-mail: wim.desmet@kuleuven.be

Additional Comments: (none)

PACS Numbers: 43.55.Br

(C) Clarivate Analytics | (C) ScholarOne, Inc., 2018. All Rights Reserved.

ScholarOne Abstracts and ScholarOne are registered trademarks of ScholarOne, Inc.

ScholarOne Abstracts Patents \#7,257,767 and \#7,263,655.

( @ScholarOneNews | System Requirements | Q. Privacy Statement | $\star$ Terms of Use

Product version number 4.16.0 (Build 70). Build date Wed Dec 12 14:48:33 EST 2018. Server ip-10-236-29-14 